\title{
Acknowledgement to Reviewers of the ISPRS International Journal of Geo-Information in 2013
}

IJGI Editorial Office, MDPI AG, Klybeckstrasse 64, CH-4057 Basel, Switzerland

Published: 25 February 2014

The editors of the ISPRS International Journal of Geo-Information would like to express their sincere gratitude to the following reviewers for assessing manuscripts in 2013:

\begin{tabular}{lll} 
Aguilar, Manuel Ángel & Chao, Anne & Dobson, Michael W. \\
Allen, Thomas & Chen, Gang & Dominguez, Javier \\
Alonso, Carlos R. García & Chen, Ruizhi & Dong, Pinliang \\
Amanatiadis, Angelos & Chen, Wang-Kun & Dwyer, Ned \\
Anand, Madhur & Chen, Xiang (Peter) & Elmongui, Hicham G. \\
Andrienko, Gennady & Cinnamon, Jonathan & Emrich, Christopher T. \\
Anthony, Edward J. & Cirillo, Marcello & Epprecht, Michael \\
Arsanjani, Jamal Jokar & Clancy, Katherine & Fabbri, Kristian \\
Ayanu, Yohannes & Coetzee, Serena & Fairbairn, David \\
Ballatore, Andrea & Coleman, D. J. & Falcão-Flor, Ana Paula \\
Barb, Adrian S. & Collin, Anthoine & Fan, Hongchao \\
Barber, Christopher & Corcoran, Padraig & Feick, Rob \\
Bartelme, Norbert & Couclelis, Helen & Ferreira, Celso M. \\
Bel, Liliane & Cromley, Ellen & Ferri, Gabriele \\
Biberhofer, Hans & Crooks, Andrew & Fisher, Pete \\
Bordogna, Gloria & Cui, Tingwei & Fisher, Rohan \\
Brando, Carmen & Da Amicis, Mattia & Foerster, Theodor \\
Canessa, Rosaline & Dall'o', Giuliano & Foka, Amalia F. \\
Cannata, Massimiliano & Davis, Ronald W. & Ford, Arianne \\
Cao, Chunxiang & De Adana, Francisco Saez & Freitas, Ramon M. \\
Castellazzi, Marie & Denham, Robert & Friese, Karl-Ingo \\
Castilla, Guillermo & Dewan, Ashraf & Fry, Richard \\
Cervi, Federico & Di Martino, Ferdinando & Fthenakis, Vasilis \\
Chang, Yang-Chi & Dilo, Arta & Fugitt, Frank \\
& & \\
\hline & &
\end{tabular}


Gaff, Holly

Ganas, Athanassios

Geri, Francesco

Gibbes, Cerian

Girres, Jean-Francois

Giuliani, Gregory

Gonçalves, Alexandre

Gonzales, Ainhoa

Granell Canut, Carlos

Granell, Carlos

Grant, Jonathan

Grastien, Alban

Guo, Ling-Zhong

Hammoudi, Karim

Han, Weiguo

Harrie, Lars

Harris, Paul

Hartemink, Nienke

Haunert, Jan Henrik

Herrera, Gerardo

Herrlich, Marc

Hofierka, J.

Hossain, Azad

Hugues, Olivier

Hummel, Karin A.

Jaen, Francisco Javier

Janelle, Donald G.

Jankowski, Piotr

Jasiewicz, JarosŁaw

Johnson, Peter

Jun, Chulmin

Kang, Emily Lei

Karimi, Hassan A.

Katsuaki, Koike

Kiffner, Christian

King, Chung-Ta

Kliment, Tomas

Knauth, Stefan

Knopper, Loren

Kosicki, Jakub Z.

Kostelnick, John

Koutsias, Nikos
Kumar, Suresh

Laue, Tim

Laughlin, Daniel

Le Combe, Steven C.

Le, Yanfen

Lee, Sang-Sun

Lentz, Erika

Leon Patino, Javier

Levin, Eugene

Leyk, Stefan

Li, Ki-Joune

Li, Linna

Li, Peijun

Li, Ruopu

Li, Songnian

Li, Weidong

Li, Wenwen

Lieske, David J.

Lin, Huei-Yung

Linsen, Lars

Lipoti, Jill

Lo, Anthony

Logsdon, Miles G.

Loidl, Martin

Looijen, Joan

Luke, Edward A.

M. Foody, Giles

Machado, Elia

Mackaness, William A.

Madden, Marguerite

Magliocca, Nicholas R.

Mannaerts, C.M.M. (Chris)

Mansourian, Ali

Marceau, Danielle J.

Marcheggiani, Ernesto

Mäs, Stephan

Matyas, C.J.

Mekni, Mehdi

Meng, Jihua

Mennis, Jeremy Laurence

Mitasova, Helena

Mittlboeck, Manfred
Molenaar, Martien

Montes, Fernando

Mooney, Peter

Moore, Antoni

Moreno-Sanchez, Rafael

Morrison-Saunders, Angus

Mottus, Matti

Mõttus, Matti

Nativi, Stefano

Nelson, Ross

Neutens, Tijs

$\mathrm{Ng}$, Joseph

Olsen, Michael J.

Omitaomu, Olufemi A.

Parmentier, Benoit

Pascual, Victor

Paulen, Roger

Pei, Ling

Peinado, Antonio M.

Ponzoni, Flavio

Poore, Barbara S.

Popescu, Voichita

Porter, Dwayne

Posa, Donato

Propastin, Pavel

Puiatti, Alessandro

Purves, Ross

Qiu, Bingwen

Quartulli, Marco

Radoux, M. Julien

Rajabifard, Abbas

Ramampiaro, Heri

Rehrl, Karl

Rey, Sergio Rey

Ripolles, Oscar

Rivera, Samuel

Robin, Marc

Ross, Lindsay

Rovere, Alessio

Ruiz, Daniel

Sanz, David

Sarapirome, Sunya 
Schade, Sven

Scheider, Simon

Schindelhauer, Christian

Schmid, Falko

Schneider, Markus

Senaratne, Hansi

Seong, J. C.

Shumchenia, Emily J

Simpson, Richard

Slingsby, Aidan

Snickars, Martin

Steinbrich, Andreas

Steiniger, Stefan

Stine, Roy

Strang, Thomas

Suárez, Jose Pablo

Tapsell, Sue

Tarantino, Eufemia

Tardif, Pierre Martin

Tarrio, Paula

Teegavarapu, Ramesh S.V.
Thapa, Rajesh Bahadur

Theilen-Willige, Barbara

Tomko, Martin

Tonn, Bruce E.

Torres, Ricardo Da S.

Torsten Prinz, Torsten

Tortosa, Leandro

Touya, Guillaume

Treuillet, Sylvie

Ureña, Manuel A.

Van Boeckel, Thomas

Van Den Berg, Frits

Van Hoesen, John

Varanka, Dalia

Verhoeven, Geert

Vogt, Brandon J.

Wagner, Bernardo

Wang, Fei

Wang, Jinfeng

Wang, JingWang, Jun

Wang, Ninghua (nathan)
Watkins, James

Wei, Yaxing

Werner, Martin

Wijaya, Arief

Wu, Changshan

$\mathrm{Wu}$, Huayi

Yamaguchi, Yasushi

Yang, Chaowei

$\mathrm{Yu}$, Bailang

Yuan, Fei

Zamiyatin, Alexander

Zamora-Izquierdo, M.A.

Zandbergen, Paul A.

Zeile, Peter

Zhang, Chuanrong

Zhang, Jixian

Zhao, Jun

Zhao, Peisheng

Zhu, Yan

Zhu, Yuanda

Zurlini, Giovanni

(C) 2014 by the authors; licensee MDPI, Basel, Switzerland. This article is an open access article distributed under the terms and conditions of the Creative Commons Attribution license (http://creativecommons.org/licenses/by/3.0/). 